\title{
Streptococcus suis in invasive human infections in Poland: clonality and determinants of virulence and antimicrobial resistance
}

\author{
A. Bojarska ${ }^{1}$ - E. Molska ${ }^{1,3}$ - K. Janas ${ }^{1,4}$ - A. Skoczyńska ${ }^{2}$ - E. Stefaniuk ${ }^{2}$. \\ W. Hryniewicz ${ }^{2}$ E. Sadowy ${ }^{1}$
}

Received: 18 December 2015 / Accepted: 25 February 2016 / Published online: 15 March 2016

(C) The Author(s) 2016. This article is published with open access at Springerlink.com

\begin{abstract}
The purpose of this study was to perform an analysis of Streptococcus suis human invasive isolates, collected in Poland by the National Reference Centre for Bacterial Meningitis. Isolates obtained from 21 patients during 20002013 were investigated by phenotypic tests, multilocus sequence typing (MLST), analysis of the TR9 locus from the multilocus variable number tandem repeat (VNTR) analysis (MLVA) scheme and pulsed-field gel electrophoresis (PFGE) of SmaI-digested DNA. Determinants of virulence and antimicrobial resistance were detected by polymerase chain reaction (PCR) and analysed by sequencing. All isolates represented sequence type 1 (ST1) and were suggested to be serotype 2 . PFGE and analysis of the TR9 locus allowed the discrimination of four and 17 types, respectively. Most of the isolates were haemolysis- and DNase-positive, and around half of them formed biofilm. Genes encoding suilysin, extracellular protein factor, fibronectin-binding protein, muramidasereleased protein, surface antigen one, enolase, serum opacity factor and pili were ubiquitous in the studied group, while
\end{abstract}

Electronic supplementary material The online version of this article (doi:10.1007/s10096-016-2616-x) contains supplementary material, which is available to authorized users.

E. Sadowy

ewasadowy@cls.edu.pl

1 Department of Molecular Microbiology, National Medicines Institute, Chełmska 30/34, 00-725 Warsaw, Poland

2 Department of Epidemiology and Clinical Microbiology, National Medicines Institute, Chełmska 30/34, 00-725 Warsaw, Poland

3 Present address: Department of Molecular Biology and Genetics, Gene Expression and Gene Medicine, C.F. Møllers Allé 3, building 1130, 422, 8000 Aarhus C, Denmark

4 Present address: Institute of Environmental Sciences, Jagiellonian University, Gronostajowa 7, 30-387 Cracow, Poland none of the isolates carried sequences characteristic for the $89 \mathrm{~K}$ pathogenicity island. All isolates were susceptible to penicillin, cefotaxime, imipenem, moxifloxacin, chloramphenicol, rifampicin, gentamicin, linezolid, vancomycin and daptomycin. Five isolates $(24 \%)$ were concomitantly non-susceptible to erythromycin, clindamycin and tetracycline, and harboured the $\operatorname{tet}(O)$ and $\operatorname{erm}(B)$ genes; for one isolate, $l s a(E)$ and $\ln u(B)$ were additionally detected. Streptococcus suis isolated in Poland from human invasive infections belongs to a globally distributed clonal complex of this pathogen, enriched in virulence markers. This is the first report of the $l s a(E)$ and $\ln u(B)$ resistance genes in S. suis.

\section{Introduction}

Streptococcus suis represents one of most important pathogens of pigs, responsible for septicaemia, meningitis, arthritis and pneumonia in newborn and young animals of this species [1]. These bacteria are also a cause of invasive diseases in humans, mainly meningitis, as well as septicaemia, endocarditis and arthritis [2]. Such infections are typically sporadic, and, in the majority of cases, occur in particular occupational groups, such as abattoir workers and butchers. Infection may also be acquired by contact with raw or undercooked meat products, traditionally consumed in the Far East of Asia [2] and, thus, S. suis should be considered a food-borne pathogen [3]. In some countries of this region, such as Vietnam, S. suis represents the most frequent cause of bacterial meningitis in adults [4]. Streptococcus suis of serotype 2 (SS2) is considered the most virulent in both humans and animals among the currently recognised 29 serotypes [2, 5]. Tonsil carriage of SS2 by healthy slaughterhouse pigs represents an important natural reservoir of this pathogen [6]. Other serotypes, sporadically isolated from humans, include 1, 4, 5, 14, 16, 21 and 24 
[2]. The threat posed by S. suis to public health was further emphasised with reports of two outbreaks in China in 1998 and 2005, involving 25 and 215 patients, and 14 and 38 deaths, respectively [7]. Streptococcal toxic shock syndrome (STSS) and high mortality of patients, observed in both outbreaks, was attributed to the presence of the $89 \mathrm{~K}$ putative pathogenicity island (89K PAI) found in strains responsible for these outbreaks [8]. Further studies identified genes encoding a two-component signal transduction system SalK/SalR, a type IV-like secretion system and a novel haemolysis-related gene hhly3, located within this element and presumably involved in the STSS development [9-11].

While $S$. suis shows quite significant variability of the general population structure, as revealed by e.g. multilocus sequence typing (MLST), human isolates belong almost exclusively to a single clonal complex (CC), $\mathrm{CC}$, with a central and likely ancestral sequence type 1 (ST1) associated with serotype 2 [12]. The most widely studied virulence-associated factors of S. suis include suilysin (Sly), extracellular factor (EF), fibronectin-binding protein (FBP), muramidase-released protein (MRP), surface antigen one (Sao), enolase (Eno), DNase (SsnA), serum opacity factor (OFS), pili and others [13-17]. Human isolates of S. suis retain susceptibility to penicillin, ceftriaxone and vancomycin, but are frequently resistant to tetracycline and erythromycin, e.g. a study in Vietnam showed prevalence rates of resistance to these compounds as high as $83 \%$ and $20 \%$, respectively [4]. Integrative conjugative elements (ICE) seem to play an important role in the transmission of resistance determinants to this species, as demonstrated by genomic studies [18]. Plasmids are observed in S. suis as well [18, 19], but their role in resistance development remains as yet little studied. Moreover, a recent study [20] has shown that S. suis is capable of developing competence for DNA uptake in a process dependent on the $\operatorname{com} R$ and $\operatorname{com} X$ gene products, thus providing another possibility for the acquisition of resistance determinants.

Recently, a meningitis case due to $S$. suis was reported in Poland [21]. The diversity of $S$. suis strains involved in invasive human infections in our country, as well as their relationships to strains from Europe and other continents, remain unknown up to now. Therefore, we aimed at performing a detailed analysis of isolates, collected by the National Reference Centre for Bacterial Meningitis (NRCBM) located at the National Medicines Institute, in the respect of their phenotypic and genotypic features.

\section{Materials and methods}

\section{Bacterial isolates and patient data}

The NRCBM started its activity in 1997, and the first $S$. suis isolate from a human invasive infection was received in 2000. Between then and the end of 2013, 21 cases of invasive infection caused by S. suis (20 cases of meningitis and one case of endocarditis) were reported to the NRCBM from 13 hospitals located throughout Poland (Table 1). Seventeen ( $81 \%$ ) patients were male; the age ranged from 28 to 67 years (average, 50 years). Overall, 19 isolates were obtained from cerebrospinal fluid (CSF) and seven isolates were from blood. For five patients, isolates were received from both blood and CSF, but only one isolate from each patient was included in the analysis. Upon receipt, all isolates were re-identified using the Rapid ID32 STREP or the VITEK II GP system (both from bioMérieux, Marcy l'Etoile, France) and stored at $-80{ }^{\circ} \mathrm{C}$.

\section{Phenotypic studies}

Antimicrobial susceptibility was tested using the broth microdilution method [22] for penicillin, cefotaxime, imipenem, erythromycin, moxifloxacin, tetracycline, chloramphenicol, rifampicin, gentamicin, linezolid and vancomycin; susceptibility to daptomycin was studied by the Etest method (bioMérieux, Marcy l'Etoile, France) and susceptibility to clindamycin by the disk diffusion method [22]. Streptococcus pneumoniae ATCC 46916 strain was used for quality control purposes. The results were interpreted following the breakpoints for viridans streptococci approved by the European Committee on Antimicrobial Susceptibility Testing (EUCAST) for penicillin, cefotaxime, imipenem, clindamycin, gentamicin and vancomycin, and the Clinical and Laboratory Standards Institute (CLSI) for of erythromycin, tetracycline, chloramphenicol, linezolid and daptomycin $[22,23]$. In the case of moxifloxacin and rifampicin, S. pneumoniae breakpoints were used [23]. Haemolysis was evaluated visually as a distinct zone around bacterial colonies on Columbia agar with $5 \%$ horse blood (bioMérieux, Marcy l'Etoile, France). The ability of isolates to form biofilm was evaluated in microtitre plates in BHI liquid medium with $0.5 \%$ glucose and with or without $2.5 \mathrm{mg} / \mathrm{ml}$ of human plasma fibrinogen (Sigma-Aldrich, St. Louis, MO, USA), followed by staining with crystal violet, as previously described [24]. A biofilm-forming clinical isolate of Enterococcus faecalis from our collection was used as a positive control. The experiment was performed in triplicate and isolates with a mean $\mathrm{OD}_{550} \geq 0.12$ were considered positive in the test. DNase activity [17] was tested by direct visual evaluation on DNase agar with Methyl Green (Becton Dickinson, Sparks, MD, USA), using Staphylococcus aureus ATCC 25923 as a positive control.

\section{DNA isolation and bacterial typing}

Total DNA was purified using the Genomic DNA Prep Plus kit following the manufacturer's instructions (A\&A Biotechnology, Gdynia, Poland). MLST was performed as 
previously described [12]; allele numbers and sequence types (STs) were assigned using the MLST database http://ssuis.mlst.net/ (accessed 17th December 2015). Pulsed-field gel electrophoresis (PFGE) analysis of SmaIdigested DNA was performed following the standard procedure [25]. The number of variable number tandem repeats (VNTR) $\left(5^{\prime} \text { GAGCA }\right)_{\mathrm{n}}$ in the TR9 locus included in the proposed multilocus VNTR analysis (MLVA) scheme [26] was established using polymerase chain reaction (PCR) amplification of TR9 and sequencing of the products. Serotype of isolates was determined using primers specific for the cps loci of serotypes 2 and 1/2 [27].

\section{Analysis of virulence determinants}

The $f b p S$, epf, eno and sly genes were detected as described by others [27-30]. The presence of orfC was investigated by PCR with primers 5'-AGATTGGGATGAACTGGTCG and 5'-AATAGCCGTATGACCTGCCA, specific for $\operatorname{orf} B$ and orfC, respectively (GenBank accession number AJ416308 [30]). The 89K PAI sequences were searched using primers $\mathrm{CH} 3$ and $\mathrm{CH} 4$, specific for unique sequences within the PAI [31]. Additionally, PCR with primers $\mathrm{CH} 1$ and $\mathrm{CH} 6$ [31], targeting sequences adjacent to this PAI, was used to exclude its presence in isolates negative in the previous reaction. The ofs and sao types were investigated as described [16, 32]. The pili genotypes were established using the published scheme employing primers specific for four pili gene clusters [15], including the sequencing of $s b p 2$. The region encompassing 1-2286 bp in $m r p$ was analysed by sequencing of the products of overlapping PCR [33], and the number of repeats in the repeat region by sizing of $\mathrm{PCR}$ products containing this region [34].

\section{Detection of antimicrobial resistance, transposon and plasmid genes}

Tetracycline resistance genes tet $(M), \operatorname{tet}(O), \operatorname{tet}(L), \operatorname{tet}(K)$, tet $(W)$, macrolide resistance genes $\operatorname{erm}(B), \operatorname{mef}(A)$ and $\operatorname{int}_{\mathrm{Tn} 916}$ were detected by PCR as described by others [35-38]. For the detection of tet(40) and the lincosamide resistance genes $l s a$ (E) and $\ln u(B)$, primer pairs tet40-up/tet $40-\mathrm{dn}$ ( $5^{\prime}$ - C TA C C T GCTGT T C C GATT T G T C and 5'-TGATGAAGGTATCACCGCAACC), lsaE-up/lsaE-dn ( $5^{\prime}$ - TATGCG TAT TCCGGCAATATAAG and 5'-AACGGCTTCCTGATGTCTTG) and lnuB-up/lnuB-dn ( $5^{\prime}$ - CGTGGGGAATT TCATTTCCTTTC and 5'-CGTTGATTCCCATCAACCATAG) were used, respectively. The linkage between genes $l s a E-\operatorname{lnu} B$ and ermB-tet $O$ was investigated with primers lsaE-up/lnuB-dn and ermB-1/ tetO-dn [36, 38], respectively. The presence of rep1 and rep2 genes, characteristic for broad-host range streptococcal and enterococcal plasmids, was tested using primers and conditions proposed in the Gram $(+)$ plasmid typing scheme

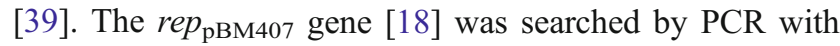
primers rep407-up/rep407-dn (5'-GTATCGCACGTA TTCCTCGTG and 5'-CATAATAGCCTTTTCCCCACGA). The $\omega-\varepsilon-\zeta$ and $r e l B E$ genes of plasmid toxin-antitoxin systems (TAS) were searched for as previously described [40]. The $r e p A$ gene, specific for ICESsu $u_{\mathrm{SC} 84}$ and ICESsu $u_{\mathrm{BM} 407^{-2}}$ [18], was detected with consensus primers repA_up/repA_dn (5'-TTAAGGTAGCCTACGCGGTTTTA and 5'-GTC GTCTCAGTTGCTTRGTCC). DNA from previously characterised clinical isolates of Streptococcus agalactiae, E. faecalis and Enterococcus faecium from our collection were used as positive controls for the detection of $\operatorname{tet}(M), \operatorname{tet}(O)$, tet $(L), \operatorname{tet}(K), \operatorname{tet}(W), \operatorname{erm}(B), \operatorname{mef}(A), \operatorname{int}_{\mathrm{Tn} 916}$, rep 1, rep $2, \omega-\varepsilon-\zeta$ and relBE. Competence-associated genes $\operatorname{com} X$ and $\operatorname{comR}$ were detected with primer pairs comX-up/comX-dn and comR-up/ comR-dn (5'-GGATCGA GATGATTGGGAAC/ 5'-CATGTGGCATACGGTCAAAC and 5'-CTGAAGTT TGATGTGCTTCGC/5'-TTTCCAAAGCCTGCTGTACCT, respectively).

\section{DNA sequence analysis}

Sequences were analysed using the Lasergene package (DNASTAR, Madison, WI, USA). New sequences found in this study were submitted to the GenBank: sao (KJ739797), mrp (KJ776426, KJ776427, KJ746998, KJ746999), ISSag3-like (KR137531).

\section{Results}

\section{Serotypes and clonality of isolates}

PCR and MLST analyses demonstrated that all isolates were positive for serotypes 2- and 1/2-specific PCR and belonged to ST1. Because no human infection due to serotype $1 / 2$ strains has yet been reported in any country [41], the PCR results strongly suggest that all the isolates are serotype 2 . PFGE analysis identified four related patterns, A1-A4 (Table 1 and Supplementary Fig. 1). The predominant pattern, A1, was characteristic of 18 isolates. Sequencing of the TR9 locus from the MLVA scheme revealed 17 variants, with 8-72 repeats.

\section{Virulence phenotypes and determinants}

Ten isolates $(48 \%)$ were able to form biofilm in the medium supplemented with fibrinogen (Table 1); no biofilm was observed in the absence of this compound. Fifteen isolates (71 \%) showed variable levels of DNase activity. With a single exception, all isolates caused various degrees of $\beta$-haemolysis of horse blood and harboured the sly gene. This single isolate 
Table 1 Selected features of human invasive isolates of Streptococcus suis isolated in Poland from 2000 to 2013

\begin{tabular}{|c|c|c|c|c|c|c|c|c|c|c|c|c|c|c|}
\hline Year & City $^{\mathrm{a}}$ & $\begin{array}{l}\text { Isolation } \\
\text { material }^{\text {b }}\end{array}$ & $\begin{array}{l}\text { Antibiotic } \\
\text { resistance } \\
\text { profile }^{c}\end{array}$ & PFGE & TR9 $^{d}$ & $\begin{array}{l}\text { Haemolysis/ } \\
\text { sly gene }{ }^{\mathrm{e}, \mathrm{f}}\end{array}$ & $\begin{array}{l}\text { Biofilm } \\
\text { formation }^{\mathrm{f}}\end{array}$ & $\begin{array}{l}\text { DNase } \\
\text { activity }^{\text {ffg }}\end{array}$ & $\begin{array}{l}\text { OFS } \\
\text { type }\end{array}$ & $\begin{array}{l}\text { sao } \\
\text { type }\end{array}$ & $\begin{array}{l}m r p, \\
\text { repeat } \\
\text { region }\end{array}$ & $\begin{array}{l}m r p, \\
\text { variable } \\
5^{\prime} \\
\text { region }^{\mathrm{h}}\end{array}$ & $\begin{array}{l}\omega- \\
\varepsilon-\zeta^{\mathrm{f}}\end{array}$ & $\begin{array}{l}r e p A \text { of } \\
\mathrm{ICESsu} u_{\mathrm{SC} 84} \\
\mathrm{ICESsu} u_{\mathrm{BM}} \\
{ }_{407^{-}}-2\end{array}$ \\
\hline 2000 & Gdańsk & $\mathrm{CSF}$ & $\mathrm{S}$ & A1 & 36 & $+++/+$ & neg & + & 1 & M & $m r p$ & wt & neg & neg \\
\hline 2003 & Gdańsk & CSF & $\mathrm{S}$ & A1 & 14 & $++1 /+$ & pos & neg & 1 & M & $m r p$ & wt & neg & neg \\
\hline 2004 & Opole & CSF/blood & $\mathrm{S}$ & A1 & 35 & $++/+$ & neg & neg & 1 & M & $m r p$ & wt & neg & neg \\
\hline 2004 & Opole & CSF & $\mathrm{S}$ & A4 & 8 & $++/+$ & pos & + & 1 & M & $m r p$ & wt & neg & neg \\
\hline 2005 & Elblagg & CSF/blood & $\mathrm{S}$ & A1 & 35 & $++/+$ & pos & ++ & 1 & M & $m r p$ & wt & neg & neg \\
\hline 2005 & $\begin{array}{l}\text { Kędzierzyn- } \\
\text { Koźle }\end{array}$ & CSF & $\mathrm{S}$ & A1 & 42 & $++/+$ & neg & neg & 1 & M & $m r p$ & wt & neg & neg \\
\hline 2006 & Nowa Sól & blood $^{\mathrm{x}}$ & $\mathrm{S}$ & A1 & 33 & $++/+$ & neg & + & 1 & M & $m r p$ & wt & neg & neg \\
\hline 2007 & Pleszew & CSF/blood & $\underline{\mathrm{ECT}}$ & A1 & 15 & $++/+$ & pos & neg & 1 & $\begin{array}{l}6 \\
\text { re- } \\
\text { pe- } \\
\text { ats }\end{array}$ & $m r p$ & $\Delta 1699$ & neg & pos \\
\hline 2008 & Gdańsk & CSF/blood & $\mathrm{S}$ & A1 & 28 & neg/neg & pos & + & 1 & $\mathrm{M}$ & $m r p$ & ins $571^{\prime}$ & neg & neg \\
\hline 2009 & Olsztyn & CSF & $\mathrm{S}$ & A1 & 15 & $++/+$ & neg & neg & 1 & M & $m r p^{\mathrm{S}}$ & wt & neg & neg \\
\hline 2010 & Brzeg & blood $^{\mathrm{y}}$ & $\mathrm{S}$ & A1 & 32 & $++/+$ & neg & ++ & 1 & M & $m r p$ & wt & neg & neg \\
\hline 2010 & Bełchatów & CSF & $\mathrm{S}$ & A1 & 34 & $++/+$ & neg & neg & 1 & M & $m r p$ & wt & neg & neg \\
\hline 2010 & Puławy & CSF & ECT & $\mathrm{A} 3$ & 19 & $++/+$ & neg & + & 1 & M & $m r p$ & wt & pos & pos \\
\hline 2010 & Koszalin & $\mathrm{CSF}$ & ECT & A1 & 26 & $++/+$ & pos & + & ISSag3 & M & $m r p$ & wt & neg & pos \\
\hline 2011 & Koszalin & CSF/blood & ECT & A2 & 72 & $+/+$ & pos & + & 1 & M & $m r p$ & wt & neg & pos \\
\hline 2011 & Chojnice & $\mathrm{CSF}$ & $\mathrm{S}$ & A1 & 29 & $++/+$ & pos & ++ & 1 & M & $m r p$ & wt & neg & neg \\
\hline 2012 & Elbląg & $\mathrm{CSF}$ & $\mathrm{S}$ & A1 & 26 & $++/+$ & pos & ++ & 1 & M & $m r p$ & wt & neg & neg \\
\hline 2012 & Wejherowo & CSF & $\mathrm{S}$ & A1 & 29 & $++/+$ & neg & ++ & 1 & M & $m r p$ & wt & neg & neg \\
\hline 2013 & Chojnice & CSF & $\mathrm{S}$ & A1 & 39 & $+/+$ & neg & ++ & 1 & M & $m r p$ & ins $571^{\prime}$ & neg & neg \\
\hline 2013 & Koszalin & CSF & ECT & A1 & 61 & $++/+$ & neg & + & 1 & M & $\Delta 162 \mathrm{bp}$ & wt & neg & pos \\
\hline 2013 & Opole & CSF & $\mathrm{S}$ & A1 & 23 & $+++/+$ & pos & + & 1 & M & $m r p$ & wt & neg & neg \\
\hline
\end{tabular}

${ }^{\text {a }}$ City of patient hospitalization

${ }^{\mathrm{b}}$ For five patients, two isolates were obtained (from CSF and blood), and both isolates shared all features tested

${ }^{\mathrm{c}} \mathrm{S}$, susceptible to all antimicrobials tested; ECT, resistance to erythromycin, clindamycin and tetracycline (isolate harbouring the $l s a(E) / l n u(B)$ genes underlined

${ }^{\mathrm{d}}$ TR9, number of repeats in the TR9 locus from the MLVA scheme

$\mathrm{e}_{+},++,+++$, weak, intermediate and strong haemolysis, respectively

${ }^{\mathrm{f}}$ pos, positive; neg, negative

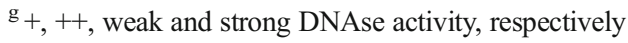

${ }^{\mathrm{h}}$ wt, wild-type sequence

${ }^{\mathrm{x}}$ Patient diagnosed with endocarditis

${ }^{\mathrm{y}}$ Patient diagnosed with meningitis

was negative in PCR for both sly and orfC. None of the isolates harboured sequences characteristic for the 89K PAI, and all isolates yielded expected $\sim 1.5 \mathrm{~kb}$ PCR product with primers specific for sequences located upstream and downstream of the PAI, further confirming its absence. All isolates carried the $f b p S$, eno, epf, sao, mrp and pili genes. The size of the PCR product for epf corresponded to the basic variant of this gene without insertions in the $3^{\prime}$ part [28]. The sao gene represented the M-type (i.e. with seven repeats) [32], with the exception of one isolate, which had six repeats. Analysis of the $3^{\prime}$ part of the mrp gene by PCR showed the presence of a single repeat for the majority (19) of isolates; one isolate had the $m r p$ S variant [34] and one had a novel $m r p$ variant, containing a 162-bp deletion in the repeat region, encompassing nt4898-5059. Partial sequencing of the variable region of $\mathrm{mrp}$ revealed the presence of two new mutations, both resulting in a translational frameshift, a two-nucleotide insertion at position 571 in two isolates and a single-nucleotide deletion at position 1699 in one case. For the remaining isolates, the sequence was $100 \%$ identical with the European variant (GenBank accession number X64450) of $m r p$ [33]. Analysis of the pili gene clusters showed that all isolates belonged to genotype A, i.e. they were positive for the $\operatorname{sr} B C D$ cluster, the $s r t E / s i p E$ genes and the $s r t F$ cluster. All isolates contained a 
single T insertion at position $798^{\prime}$ of the $s b p 2$ gene, which split the $s b p 2$ open reading frame (ORF) into $s b p 2^{\prime}$ and $s b p 2^{\prime \prime}$ [15]. All isolates carried the type 1 ofs gene, with the exception of one isolate, for which partial sequencing revealed the presence of the ISSag3-like insertion sequence from $S$. agalactiae [42] (97\% identity at the nucleotide level) at nt1165, in the same orientation as the ofs gene.

\section{Antimicrobial susceptibility profiles of isolates, resistance and competence determinants}

All isolates were susceptible to penicillin, cefotaxime, imipenem, moxifloxacin, chloramphenicol, rifampicin, gentamicin, linezolid, vancomycin and daptomycin. Five isolates (24\%) were concomitantly resistant to tetracycline, erythromycin and clindamycin (Table 1). Resistant isolates had three different PFGE patterns (A1, A2, A3) and they all harboured the $\operatorname{tet}(O)$ and $\operatorname{erm}(B)$ genes; other tetracycline and macrolide resistance genes, such as tet $(M), \operatorname{tet}(40), \operatorname{tet}(L), \operatorname{tet}(K), \operatorname{tet}(W)$ and $m e f(A)$ were not detected. None of the isolates was positive for the ermB-tetO linkage, characteristic for the ICESsu $u_{\mathrm{BM} 407^{-}} 2$ [18]. Additionally, one isolate was positive for the $l s a(E)$ gene and the $\ln u(B)$ gene (in both cases verified by sequencing). As demonstrated by PCR with primers specific for the two genes, $\ln u(B)$ was located directly downstream of $l s a(E)$. All 21 isolates tested negative for the presence of transposon- and plasmid-specific genes, such as int $_{T n 916}$, rep1, rер 2, rер pBM407 $_{4}$ and relBE; a single resistant isolate carried the $\omega-\varepsilon-\zeta$ genes of TAS. The rep $A$ gene, characteristic for ICESsu $u_{\mathrm{SC} 84}$ and ICESsu $u_{\mathrm{BM} 407}-2$, occurred exclusively among five resistant isolates. All isolates were positive for the $\operatorname{com} R$ and $\operatorname{com} X$ competence genes.

\section{Discussion}

Streptococcus suis is currently emerging as an important zoonotic pathogen in humans, especially in some regions of the world. The aim of our study was to provide extensive characterisation of isolates of this pathogen observed in Poland since 2000. Most of the patients affected by S. suis infection were male and middle-aged; the patient's profession is not routinely reported to the NRCBM, but for two patients (a farmer and a butcher), infection was very likely caused by occupational exposure. Misidentification is considered a common cause for the underestimation of rates of invasive infections caused by $S$. suis [2]. In our study, isolates were reported to the reference laboratory as $S$. suis in only 11 cases (52\%); five isolates were reported as Streptococcus spp. and two as Streptococcus bovis, while the remaining three isolates were misidentified as S. agalactiae, Streptococcus anginosus and Streptococcus sanguinis.
On the basis of the serotype-specific PCR results and epidemiological information reported so far [41], all studied isolates were determined as serotype 2 , which is most frequently observed in infections of humans [2, 41]. It has to be noted, however, that DNA-based methods do not allow discerning between serotypes 2 and 1/2, whose cps loci do not differ by any serotype-specific genes and whose sequences are almost identical [43]. Although strains of serotype $1 / 2$ have not been reported from human infections [41], the presence of isolates with this serotype cannot be completely excluded in our collection due to the limitations of the method used. MLST uniformly identified all isolates as ST1. Isolates of this ST and its variants have been reported from human invasive infections worldwide, although STs from other clonal complexes have also been described, including, for example, ST20 in France and the Netherlands, STs 25 and 28 in Thailand and Japan, and ST25 in the United States (summarised in [41]). As shown recently in a Dutch study [44], the diversity of human isolates of $S$. suis appears generally much more limited in comparison to strains circulating in pigs in the same geographic area and a similar time span, and the vast majority of STs and serotypes found in pigs, a natural reservoir of this pathogen, have never been isolated from humans [41]. Our PFGE analysis discerned four different profiles and we additionally applied MLVA, which was suggested as a typing method allowing additional discrimination of strains, e.g. during an outbreak [26]. In the case of ST1, variability was reported only for the TR9 locus, which was selected for in our analyses. These yielded 17 variants with an even bigger range of the repeat number (8-72) than observed in the original study [26]. Such high diversity of a presumably quickly evolving typing marker is consistent with the sporadic character of all cases of human infections from which our isolates were obtained.

Biofilms play an important role in reducing bacterial susceptibility to antimicrobials and clearance by the host immune system. Formation of biofilm by $S$. suis required the presence of fibrinogen in the culture medium [24], as also seen in our study. We observed various levels of biofilm formation among our isolates, which was also reported by others for SS2 [45]. Some of our isolates lacked detectable DNase activity, in contrast to the findings of Haas and co-workers, who reported all ten ST1 strains in their study as DNase-positive [17]. Almost all isolates demonstrated haemolytic phenotype, associated with the presence of the sly gene. Sly, a pore-forming toxin, plays an important role in the pathogenesis of $S$. suis infection [46] and several studies reported all ST1 human isolates as positive for sly [47-50], in contrast to representatives of other clonal groups found in humans [49] and isolates from non-invasive infections and carriage in pigs [30, 34]. This gene is typically well conserved in S. suis and loss of haemolytic activity is usually due to the replacement of sly by orfC encoding a product of unknown function [30]; however, one of the isolates in the current study lacked both sly 
and $\operatorname{orfC}$. Such isolates were also observed by others [48] and our searches of the available genomic sequences of $S$. suis in the GenBank (as of 29th January 2016) revealed that a single strain YS56 (GenBank accession number ALMY01000022) was negative for these two genes in the corresponding position of its genome.

The isolates analysed in our study carried genes of several virulence-associated factors other than sly, such as mrp, epf, $f b p S$, eno, sao and ofs. The basic variant of the epf gene appears to be, similarly to $s l y$, a marker specific for invasive ST1 strains [47-50], while $m r p, f b p S$, eno, sao and ofs appear to be much more common in the whole $S$. suis population [29, 32, 47-51]. In agreement with other observations, our analysis also revealed variation in the genes of some of these virulence-associated determinants, including novel alleles of $m r p$, sao and ofs. The $m r p$ gene with a single 411-bp repeat in its $3^{\prime}$ part, most common among our isolates, is typical for ST1; a shorter version, $m r p^{\mathrm{S}}$, present in one isolate, was also observed for this ST [49]. Larger variants of $m r p$ occur among representatives of other CCs associated with serotype 2, such as ST29 [49] and other serotypes [34]. Three isolates from our collection harboured new indel mutations in $m r p$, preventing the full-length Mrp protein synthesis. Such mutations are relatively common in S. suis [33], and isolates positive for the gene but negative for the protein expression are frequently observed $[33,34,50]$. Variability in the $m r p$ gene may be associated with a selective pressure from the immunological system of the host [34]. The sao-M (seven repeats), observed in our study for almost all isolates, is the most common variant among various serotypes of $S$. suis [32], and type 1 ofs characteristic for all but one isolate is typical for ST1 [16]. In our study, all isolates belonged to the genotype A of pili and harboured the characteristic frame-shift mutation in sbp2. At least four different pili loci exist in S. suis, and the combination of presence/absence of particular genes allowed distinguishing 12 genotypes, with genotype A being characteristic for ST1 isolates from human infections and diseased pigs [15]. We did not detect sequences specific for the $89 \mathrm{~K}$ candidate PAI, found in highly virulent strains involved in two outbreaks in China and, as yet, not observed anywhere else [31]. In summary, inclusion of all the study isolates into ST1 and SS2, together with the observed high number of established and putative virulence factors, are consistent with the features of a specific genetic cluster of $S$. suis, associated with human meningitis [52], described also as the epidemic and highly virulent $(\mathrm{E} / \mathrm{HV})$ group, showing resistance to phagocytosis in vivo, thus allowing bacteria persistence at high concentrations in the animal mouse model, a pre-requisite for the development of an inflammatory reaction in the host [53].

Importantly, the investigated isolates of $S$. suis from our collection retained susceptibility to agents recommended in Poland for empirical therapy of community-acquired bacterial meningitis in adults, such as cefotaxime and vancomycin (http://www.koroun.edu.pl, accessed 17th December 2015). As yet, no resistance to these compounds has been reported for $S$. suis isolated from humans [54-56]. We observed concomitant resistance to erythromycin and tetracycline for $24 \%$ of isolates. Resistance to tetracycline appears to be common, or even very common, in S. suis from human infections, reaching $100 \%$ among 114 strains of ST7 in China [54], $100 \%$ among 33 isolates in Hong Kong [55] and $89 \%$ among 175 isolates in Vietnam, with a clear increase over the period 1997-2008 [56]. Resistance rates to erythromycin were $21 \%$ and $22 \%$ in Hong Kong and Vietnam, respectively $[55,56]$, similar to our observations. The acquisition of resistance determinants by $S$. suis occurs chiefly in its animal host under the selective pressure exerted by the use of antimicrobial agents in animal production. In Poland, according to the European Surveillance of Veterinary Antimicrobial Consumption (ESVAC) report for 2011, the sales of lincosamides, macrolides and tetracyclines for food-producing animals amounted to 4.1, 25.6 and 179.6 tonnes, respectively [57], and resistance to erythromycin and tetracycline among pig isolates reached $31 \%$ and $64 \%$, respectively, in 2004 [58]. In our collection, isolates resistant to tetracycline and erythromycin/clindamycin carried the $\operatorname{tet}(O)$ and $\operatorname{erm}(B)$ genes. The presence of $\operatorname{tet}(O)$ was reported for a small number of human isolates from Europe and North America [54]. The $\operatorname{tet}(M)$ gene, associated with $\operatorname{Tn} 916$, was the most widespread determinant of tetracycline resistance in human S. suis in China and Vietnam [54, 56]. In Vietnam, tet $(O)$ was the second most common gene responsible for tetracycline resistance; $\operatorname{tet}(L)$ and $\operatorname{tet}(W)$ were also observed in that study [56]. The $\operatorname{erm}(B)$ gene was present in $95 \%$ of erythromycin-resistant human S. suis in Vietnam [56], while in Hong Kong, most of the isolates carried mef(A) [55]. Thus, there are obvious differences in the local epidemiology of tetracycline and erythromycin resistance genes among human $S$. suis. Additionally, one of the resistant isolates in our collection carried the $l s a(E)$ and $\operatorname{lnu}(B)$ genes. The $l s a(E)$ gene encodes an $\mathrm{ABC}$ transporter that confers resistance to lincosamides, streptogramin $\mathrm{A}$ and pleuromutilins $\left(\mathrm{LS}_{\mathrm{A}} \mathrm{P}\right.$ phenotype) by efflux of the drugs from the bacterial cell [59], while $\ln u(B)$ (formerly $\operatorname{lin} B$ ) inactivates lincosamides by adenylation [60]. These two genes are found in several Gram(+) bacteria, e.g. E. faecalis (AF408195), the C2944 strain of $S$. aureus, the SGB76 strain of $S$. agalactiae, the pDX5 plasmid of E. faecium and the Ery-11 strain of the pig pathogen Erysipelothrix rhusiopathiae [61-64], but, to our knowledge, have not yet been reported in S. suis. Our search of genomic sequences of 375 pig and human isolates of S. suis [65], deposited at the European Nucleotide Archive (http:// www.ebi.ac.uk/ena, accessed 2nd February 2016), revealed the presence of $l s a(E)$ and $\ln u(B)$ in four pig 
isolates, associated with serotype 2 from Vietnam and in two pig isolates of serotypes 7 and 14 from the United Kingdom, suggesting independent acquisition of these resistance genes by different clones in various parts of the world. The macrolide, lincosamide and tetracycline resistance determinants detected among isolates from our collection were likely carried by $S$. suis-specific ICEs [18] , consistent with the concomitant presence of their repA gene and absence of genes characteristic for other mobile elements, such as Tn916 and broad-host plasmids.

In conclusion, we performed a detailed phenotypic and genotypic analysis of $S$. suis isolates obtained in Poland from invasive human infections. While all these isolates belonged to a single clonal complex associated with considerable morbidity and mortality in humans worldwide, diversification of this complex was observed, including the presence of novel variants of virulence determinants and the acquisition of antimicrobial resistance genes. The human invasive $S$. suis infections in Poland very likely remain underestimated, considering the fact that, in 2012 , the number of professionally active farmers amounted to 2.1 million and pig livestock exceeded 10 million heads (http://www.stat.gov.pl, accessed 17th December 2015), which indicates an existence of a significant at-risk human population and a need for improved surveillance of invasive human infections, caused by this pathogen in our country.

Acknowledgements We thank all Polish microbiologists who sent S. suis isolates to our laboratory and Stephen Murchan for the critical reading of the manuscript. This publication made use of the Streptococcus suis MLST website (http://ssuis.mlst.net/) hosted at Imperial College of the University of Oxford and funded by the Wellcome Trust. Parts of this study were presented at the 24th European Congress of Clinical Microbiology and Infectious Diseases (ECCMID), 10-13th May 2014, Barcelona, Spain.

Compliance with ethical standards Isolates were obtained as part of the routine activity of the NRCBM and were analysed anonymously in a retrospective manner. Ethical approval and informed consent were, thus, not required.

Conflict of interest The authors declare that they have no conflict of interest.

Funding This work was supported by the grant NZ2/01945 from Narodowe Centrum Nauki (NCN), Poland.

Open Access This article is distributed under the terms of the Creative Commons Attribution 4.0 International License (http:// creativecommons.org/licenses/by/4.0/), which permits unrestricted use, distribution, and reproduction in any medium, provided you give appropriate credit to the original author(s) and the source, provide a link to the Creative Commons license, and indicate if changes were made.

\section{References}

1. Staats JJ, Feder I, Okwumabua O, Chengappa MM (1997) Streptococcus suis: past and present. Vet Res Commun 21:381-407

2. Huong VT, Ha N, Huy NT et al (2014) Epidemiology, clinical manifestations, and outcomes of Streptococcus suis infection in humans. Emerg Infect Dis 20:1105-1114. doi:10.3201/eid2007. 131594

3. Ferrando ML, de Greeff A, van Rooijen WJ et al (2015) Hostpathogen interaction at the intestinal mucosa correlates with zoonotic potential of Streptococcus suis. J Infect Dis 212:95-105. doi: 10.1093/infdis/jiu813

4. Mai NT, Hoa NT, Nga TV et al (2008) Streptococcus suis meningitis in adults in Vietnam. Clin Infect Dis 46:659-667. doi:10.1086/ 527385

5. le Tien HT, Nishibori T, Nishitani Y, Nomoto R, Osawa R (2013) Reappraisal of the taxonomy of Streptococcus suis serotypes 20, 22, 26, and 33 based on DNA-DNA homology and sodA and recN phylogenies. Vet Microbiol 162:842-849. doi:10.1016/j.vetmic. 2012.11.001

6. Ngo TH, Tran TB, Tran TT et al (2011) Slaughterhouse pigs are a major reservoir of Streptococcus suis serotype 2 capable of causing human infection in southern Vietnam. PLoS One 6:e17943. doi:10. 1371/journal.pone.0017943

7. Yu H, Jing H, Chen Z et al (2006) Human Streptococcus suis outbreak, Sichuan, China. Emerg Infect Dis 12:914-920

8. Chen C, Tang J, Dong W et al (2007) A glimpse of streptococcal toxic shock syndrome from comparative genomics of S. suis 2 Chinese isolates. PLoS One 2:e315

9. Li M, Wang C, Feng Y et al (2008) SalK/SalR, a two-component signal transduction system, is essential for full virulence of highly invasive Streptococcus suis serotype 2. PLoS One 3:e2080. doi:10. 1371/journal.pone.0002080

10. Zhao Y, Liu G, Li S et al (2011) Role of a type IV-like secretion system of Streptococcus suis 2 in the development of streptococcal toxic shock syndrome. J Infect Dis 204:274-281. doi:10.1093/ infdis/jir261

11. Zheng JX, Li Y, Zhang H, Fan HJ, Lu CP (2013) Identification and characterization of a novel hemolysis-related gene in Streptococcus suis serotype 2. PLoS One 8:e74674. doi:10.1371/journal.pone. 0074674

12. King SJ, Leigh JA, Heath PJ, Luque I, Tarradas C, Dowson CG, Whatmore AM (2002) Development of a multilocus sequence typing scheme for the pig pathogen Streptococcus suis: identification of virulent clones and potential capsular serotype exchange. J Clin Microbiol 40:3671-3680

13. Feng Y, Zhang H, Ma Y, Gao GF (2010) Uncovering newly emerging variants of Streptococcus suis, an important zoonotic agent. Trends Microbiol 18:124-131. doi:10.1016/j.tim.2009.12.003

14. Fittipaldi N, Segura M, Grenier D, Gottschalk M (2012) Virulence factors involved in the pathogenesis of the infection caused by the swine pathogen and zoonotic agent Streptococcus suis. Future Microbiol 7:259-279. doi:10.2217/fmb.11.149

15. Takamatsu D, Nishino H, Ishiji T et al (2009) Genetic organization and preferential distribution of putative pilus gene clusters in Streptococcus suis. Vet Microbiol 138:132-139. doi:10.1016/j. vetmic.2009.02.013

16. Takamatsu D, Osaki M, Tharavichitkul P, Takai S, Sekizaki T (2008) Allelic variation and prevalence of serum opacity factor among the Streptococcus suis population. J Med Microbiol 57: 488-494. doi:10.1099/jmm.0.47755-0

17. Haas B, Bonifait L, Vaillancourt K, Charette SJ, Gottschalk M, Grenier D (2014) Characterization of DNase activity and gene in Streptococcus suis and evidence for a role as virulence factor. BMC Res Notes 7:424. doi:10.1186/1756-0500-7-424 
18. Holden MT, Hauser H, Sanders M et al (2009) Rapid evolution of virulence and drug resistance in the emerging zoonotic pathogen Streptococcus suis. PLoS One 4:e6072

19. Cantin M, Harel J, Higgins R, Gottschalk M (1992) Antimicrobial resistance patterns and plasmid profiles of Streptococcus suis isolates. J Vet Diagn Invest 4:170-174

20. Zaccaria E, van Baarlen P, de Greeff A, Morrison DA, Smith H, Wells JM (2014) Control of competence for DNA transformation in Streptococcus suis by genetically transferable pherotypes. PLoS One 9:e99394. doi:10.1371/journal.pone.0099394

21. Zalas-Wiecek P, Michalska A, Grabczewska E, Olczak A, Pawlowska M, Gospodarek E (2013) Human meningitis caused by Streptococcus suis. J Med Microbiol 62:483-485. doi:10. 1099/jmm.0.046599-0

22. Clinical and Laboratory Standards Institute (CLSI) (2010) Performance standards for antimicrobial susceptibility testing; Twentieth informational supplement. CLSI document M100-S20. CLSI, Wayne, PA

23. European Committee on Antimicrobial Susceptibility Testing (EUCAST) (2014) Breakpoint tables for interpretation of MICs and zone diameters. Version 4.0. Available online at: http://www. eucast.org/fileadmin/src/media/PDFs/EUCAST files/Breakpoint tables/Breakpoint table_v 4.0.pdf. Accessed 17th December $201 \overline{5}$

24. Bonifait L, Grignon L, Grenier D (2008) Fibrinogen induces biofilm formation by Streptococcus suis and enhances its antibiotic resistance. Appl Environ Microbiol 74:4969-4972. doi:10.1128/ AEM.00558-08

25. Berthelot-Hérault F, Marois C, Gottschalk M, Kobisch M (2002) Genetic diversity of Streptococcus suis strains isolated from pigs and humans as revealed by pulsed-field gel electrophoresis. J Clin Microbiol 40:615-619

26. Li W, Ye C, Jing H et al (2010) Streptococcus suis outbreak investigation using multiple-locus variable tandem repeat number analysis. Microbiol Immunol 54:380-388. doi:10.1111/j.1348-0421. 2010.00228.x

27. Zhu Y, Tan Z, Zhu L et al (2008) Streptococcus suis serotype 2 caused streptococcal toxic shock syndrome (STSS) in a patient. J Nanjing Med Univ 22:313-316

28. Wisselink HJ, Reek FH, Vecht U, Stockhofe-Zurwieden N, Smits MA, Smith HE (1999) Detection of virulent strains of Streptococcus suis type 2 and highly virulent strains of Streptococcus suis type 1 in tonsillar specimens of pigs by PCR. Vet Microbiol 67:143-157

29. Esgleas M, Li Y, Hancock MA, Harel J, Dubreuil JD, Gottschalk M (2008) Isolation and characterization of alpha-enolase, a novel fibronectin-binding protein from Streptococcus suis. Microbiology 154:2668-2679. doi:10.1099/mic.0.2008/017145-0

30. King SJ, Heath PJ, Luque I, Tarradas C, Dowson CG, Whatmore AM (2001) Distribution and genetic diversity of suilysin in Streptococcus suis isolated from different diseases of pigs and characterization of the genetic basis of suilysin absence. Infect Immun 69:7572-7582

31. Schmid S, O'Connor M, Okwumabua O (2011) The pathogenicity island-like DNA segment associated with Chinese outbreak strain of Streptococcus suis serotype 2 is absent in the United States isolates. Int J Mol Epidemiol Genet 2:56-60

32. Feng Y, Zheng F, Pan X et al (2007) Existence and characterization of allelic variants of Sao, a newly identified surface protein from Streptococcus suis. FEMS Microbiol Lett 275:80-88

33. Fittipaldi N, Fuller TE, Teel JF et al (2009) Serotype distribution and production of muramidase-released protein, extracellular factor and suilysin by field strains of Streptococcus suis isolated in the United States. Vet Microbiol 139:310 317. doi:10.1016/j.vetmic.2009.06.024
34. Silva LM, Baums CG, Rehm T et al (2006) Virulence-associated gene profiling of Streptococcus suis isolates by PCR. Vet Microbiol 115:117-127

35. Doherty N, Trzcinski K, Pickerill P, Zawadzki P, Dowson CG (2000) Genetic diversity of the tet $(M)$ gene in tetracyclineresistant clonal lineages of Streptococcus pneumoniae. Antimicrob Agents Chemother 44:2979-2984

36. Trzcinski K, Cooper BS, Hryniewicz W, Dowson CG (2000) Expression of resistance to tetracyclines in strains of methicillinresistant Staphylococcus aureus. J Antimicrob Chemother 45:763770

37. Aminov RI, Garrigues-Jeanjean N, Mackie RI (2001) Molecular ecology of tetracycline resistance: development and validation of primers for detection of tetracycline resistance genes encoding ribosomal protection proteins. Appl Environ Microbiol 67:22-32. doi:10.1128/AEM.67.1.22-32.2001

38. Sutcliffe J, Grebe T, Tait-Kamradt A, Wondrack L (1996) Detection of erythromycin-resistant determinants by PCR. Antimicrob Agents Chemother 40:2562-2566

39. Jensen LB, Garcia-Migura L, Valenzuela AJ, Løhr M, Hasman H, Aarestrup FM (2010) A classification system for plasmids from enterococci and other Gram-positive bacteria. J Microbiol Methods 80:25-43. doi:10.1016/j.mimet.2009.10.012

40. Moritz EM, Hergenrother PJ (2007) Toxin-antitoxin systems are ubiquitous and plasmid-encoded in vancomycin-resistant enterococci. Proc Natl Acad Sci U S A 104:311-316

41. Goyette-Desjardins G, Auger JP, Xu J, Segura M, Gottschalk M (2014) Streptococcus suis, an important pig pathogen and emerging zoonotic agent-an update on the worldwide distribution based on serotyping and sequence typing. Emerg Microbes Infect 3:e45. doi: 10.1038/emi.2014.45

42. Liu G, Zhang W, Lu C (2012) Complete genome sequence of Streptococcus agalactiae GD201008-001, isolated in China from Tilapia with meningoencephalitis. J Bacteriol 194:6653. doi:10. 1128/JB.01788-12

43. Okura M, Takamatsu D, Maruyama F et al (2013) Genetic analysis of capsular polysaccharide synthesis gene clusters from all serotypes of Streptococcus suis: potential mechanisms for generation of capsular variation. Appl Environ Microbiol 79:2796-2806. doi: 10.1128/AEM.03742-12

44. Schultsz C, Jansen E, Keijzers W et al (2012) Differences in the population structure of invasive Streptococcus suis strains isolated from pigs and from humans in The Netherlands. PLoS One 7: e33854. doi:10.1371/journal.pone.0033854

45. Dawei G, Liping W, Chengping L (2012) In vitro biofilm forming potential of Streptococcus suis isolated from human and swine in China. Braz J Microbiol 43:993-1004. doi:10.1590/S1517838220120003000021

46. He Z, Pian Y, Ren Z et al (2014) Increased production of suilysin contributes to invasive infection of the Streptococcus suis strain 05ZYH33. Mol Med Rep 10:2819-2826. doi:10.3892/mmr.2014. 2586

47. Takamatsu D, Wongsawan K, Osaki M et al (2008) Streptococcus suis in humans, Thailand. Emerg Infect Dis 14:181-183. doi:10. 3201/eid1401.070568

48. Fittipaldi $\mathrm{N}, \mathrm{Xu}$ J, Lacouture $\mathrm{S}$ et al (2011) Lineage and virulence of Streptococcus suis serotype 2 isolates from North America. Emerg Infect Dis 17:2239-2244. doi:10.3201/eid1712.110609

49. Kerdsin A, Dejsirilert S, Puangpatra P et al (2011) Genotypic profile of Streptococcus suis serotype 2 and clinical features of infection in humans, Thailand. Emerg Infect Dis 17:835-842. doi:10. 3201/eid1705.100754

50. de Greeff A, Wisselink HJ, de Bree FM et al (2011) Genetic diversity of Streptococcus suis isolates as determined by comparative genome hybridization. BMC Microbiol 11:161. doi:10.1186/ 1471-2180-11-161 
51. Dong W, Ma J, Zhu Y et al (2015) Virulence genotyping and population analysis of Streptococcus suis serotype 2 isolates from China. Infect Genet Evol 36:483-489. doi:10.1016/j.meegid.2015. 08.021

52. Chen C, Zhang W, Zheng H et al (2013) Minimum core genome sequence typing of bacterial pathogens: a unified approach for clinical and public health microbiology. J Clin Microbiol 51:25822591. doi:10.1128/JCM.00535-13

53. Zheng H, Lan R, Zheng X et al (2014) Comparative genomic hybridization identifies virulence differences in Streptococcus suis. PLoS One 9:e87866. doi:10.1371/journal.pone.0087866

54. Ye C, Bai X, Zhang J et al (2008) Spread of Streptococcus suis sequence type 7, China. Emerg Infect Dis 14:787-791. doi:10. 3201/eid1405.070437

55. Chu YW, Cheung TK, Chu MY et al (2009) Resistance to tetracycline, erythromycin and clindamycin in Streptococcus suis serotype 2 in Hong Kong. Int J Antimicrob Agents 34:181-182. doi:10. 1016/j.ijantimicag.2009.01.007

56. Hoa NT, Chieu TT, Nghia HD et al (2011) The antimicrobial resistance patterns and associated determinants in Streptococcus suis isolated from humans in Southern Vietnam, 1997-2008. BMC Infect Dis 11:6. doi:10.1186/1471-2334-11-6

57. European Medicines Agency (EMA) (2013) Sales of veterinary antimicrobial agents in 25 EU/EEA countries in 2011. Third ESVAC report. EMA/236501/2013. Available online at: http:// www.ema.europa.eu/docs/en_GB/document library/Report/2013/ 10/WC500152311.pdf. Accessed 17th December 2015

58. Hendriksen RS, Mevius DJ, Schroeter A et al (2008) Occurrence of antimicrobial resistance among bacterial pathogens and indicator bacteria in pigs in different European countries from year 20022004: the ARBAO-II study. Acta Vet Scand 50:19. doi:10.1186/ 1751-0147-50-19
59. Wendlandt S, Lozano C, Kadlec K et al (2013) The enterococcal $\mathrm{ABC}$ transporter gene $l s a(E)$ confers combined resistance to lincosamides, pleuromutilins and streptogramin A antibiotics in methicillin-susceptible and methicillin-resistant Staphylococcus aureus. J Antimicrob Chemother 68:473-475. doi:10.1093/jac/ dks398

60. Bozdogan B, Berrezouga L, Kuo MS et al (1999) A new resistance gene, $\operatorname{lin} B$, conferring resistance to lincosamides by nucleotidylation in Enterococcus faecium HM1025. Antimicrob Agents Chemother 43:925-929

61. Lozano C, Aspiroz C, Sáenz Y et al (2012) Genetic environment and location of the $\ln u(A)$ and $\ln u(B)$ genes in methicillin-resistant Staphylococcus aureus and other staphylococci of animal and human origin. J Antimicrob Chemother 67:2804-2808. doi:10.1093/ $\mathrm{jac} / \mathrm{dks} 320$

62. Montilla A, Zavala A, Cáceres Cáceres R et al (2014) Genetic environment of the $\ln u(B)$ gene in a Streptococcus agalactiae clinical isolate. Antimicrob Agents Chemother 58:5636-5637. doi:10. 1128/AAC.02630-14

63. Wang XM, Li XS, Wang YB et al (2015) Characterization of a multidrug resistance plasmid from Enterococcus faecium that harbours a mobilized bcrABDR locus. J Antimicrob Chemother 70: 609-611. doi:10.1093/jac/dku416

64. Zhang A, Xu C, Wang $\mathrm{H}$ et al (2015) Presence and new genetic environment of pleuromutilin-lincosamidestreptogramin A resistance gene lsa(E) in Erysipelothrix rhusiopathiae of swine origin. Vet Microbiol 177:162-167. doi:10.1016/j.vetmic.2015.02.014

65. Weinert LA, Chaudhuri RR, Wang J et al (2015) Genomic signatures of human and animal disease in the zoonotic pathogen Streptococcus suis. Nat Commun 6:6740. doi:10.1038/ ncomms 7740 